\title{
Levothyroxine supplementation for obesity-associated thyroid dysfunction in children: a prospective, randomized, case control study
}

Suplementacja lewotyroksyną dysfunkcji tarczycy związanej z otyłością u dzieci: prospektywne, randomizowane badanie kliniczno-kontrolne

\author{
Sugantha Kumar, Devi Dayal, Savita Verma Attri, Atul Gupta, Anil Kumar Bhalla
}

Department of Pediatrics, Postgraduate Institute of Medical Education and Research, Chandigarh, India

\begin{abstract}
Introduction: The specific treatment of thyroid dysfunction associated with simple obesity (SO) in children is controversial.

Material and methods: Fifty-one children having SO (BMI $\geq 2$ Z-score) aged 6-12 years with elevation of serum thyroid stimulating hormone (TSH) (4-10 mIU/l) with or without alterations in serum triiodothyronine (T3) or thyroxine (T4) were randomised to receive either routine weight management intervention (group $1, n=25)$ or additional low dose levothyroxine $(\sim 0.5 \mu \mathrm{g} / \mathrm{kg} / \mathrm{day})(\mathrm{group} 2$, $n=26$ ) and followed up at 3-month intervals for 6 months.

Results: The mean BMI Z-scores decreased in both groups from baseline to 3 and 6-month time points (group 1, $3.69 \pm 1.31$ to $3.24 \pm 1.45$ to $2.84 \pm 1.46, p=0.03$ and group $2,4.31 \pm 2.11$ to $4.08 \pm 1.92$ to $3.50 \pm 1.93, p=0.02$ ). However, the decrease was similar in the 2 groups $(-0.80 \pm 0.77$ vs $-0.97 \pm 0.69, p=0.19)$. All children had isolated hyperthyrotropinemia. The mean TSH levels decreased from baseline to endpoint in group 1 (5.51 \pm 1.0 to $4.14 \pm 1.0$ to $4.04 \pm 1.1 \mathrm{mIU} / \mathrm{l}$, mean change $-1.44 \pm 1.20$, $p=0.001)$ and in group $2(5.89 \pm 1.10$ to $5.89 \pm 1.10$ to $3.89 \pm 1.48 \mathrm{mIU} / \mathrm{l}$, mean change $-1.93 \pm 1.15, p=0.001)$. The decrease in TSH levels was also similar in the 2 groups $(p=0.69)$. TSH normalized in $48 \%$ and $57.6 \%$ in group 1 and group 2 respectively. The decrease in total cholesterol and triglycerides was also similar in the 2 groups.

Conclusions: Addition of levothyroxine to weight management interventions has no beneficial effect on BMI reduction as well as parameters of lipid and thyroid profiles, and should not be prescribed to children with obesity associated thyroid dysfunction.

Key words:

exogenous obesity, obesity associated thyroid dysfunction, isolated hyperthyrotropinemia, subclinical hypothyroidism, levothyroxine, children.
\end{abstract}




\section{Introduction}

Simple obesity (SO) in children is associated with several metabolic and hormonal disturbances which contribute to the risk of developing chronic lifestyle-related diseases in later life $[1,2]$. While the metabolic problems occur due to the excess of adipose tissue that works as an endocrine organ, the hormonal disturbances are attributed to the changes in the plasma concentrations, secretory patterns and clearance of various hormones in SO [3]. Of the several hormonal disturbances in $\mathrm{SO}$, thyroid dysfunction is the most commonly reported with a prevalence that ranges from 9.2 to $31.4 \%$ in different studies $[4,5]$. The most common abnormality of thyroid function in children with $\mathrm{SO}$ is an isolated hyperthyrotropinemia (IsHT) also known as subclinical hypothyroidism ( $\mathrm{SCH}$ ) followed by minor changes in the ratios of triiodothyronine (T3) and thyroxine $(\mathrm{T} 4)[3,4]$. Of the several underlying mechanisms proposed to explain thyroid dysfunction, the majority is poorly understood [4]. These include an adaptive response to increase energy expenditure, increased leptin production by adipose tissue, resistance to thyroid hormones and insulin, increased concentrations of inflammatory cytokines and thyroid autoimmunity [4]. None of these mechanisms, however, explains the thyroid dysfunction completely and hence the clinical implications remain non-absolute and specific therapy with levothyroxine is controversial $[3,4]$. Mild $\mathrm{SCH}$, unless associated with Hashimoto's thyroiditis, is frequently considered benign in pediatric population $[6,7]$ and therapy is recommended only if the thyroid stimulating hormone $(\mathrm{TSH})$ remains consistently elevated [8-10]. Nevertheless, several recent studies have identified IsHT as an important risk factor for the development and progression of cardiovascular disease and nonalcoholic fatty liver disease (NAFLD) in obese children and adolescents [11-13]. In addition, the current understanding that SO associated thyroid dysfunction may lead to other metabolic and hormonal alterations make an argument for its treatment with levothyroxine $[3,4]$. Physicians often prescribe levothyroxine to these children alongwith other interventions believing that this may help in normalisation of body weight or overcome the difficulties in maintaining the weight loss [3, 14].

The data on levothyroxine supplementation for thyroid dysfunction in children with SO is scarce. Clinical studies have not shown beneficial effects of levothyroxine supplementation on body weight $[15,16]$. Additionally, use of levothyroxine may lead to subclinical hyperthyroidism in some patients $[16,17]$. Several studies have in fact shown normalization of thyroid dysfunction with weight loss only [18-22]. A majority of these studies were either small in size, retrospective in design, and included diverse patient age groups and suggested further testing of the usefulness of additional levothyroxine in pediatric SO $[3,14]$. We thus aimed to study the effect of levothyroxine supplementation on SO related thyroid dysfunction in children.

\section{Material and methods}

\section{Enrolment of study subjects}

Children with SO aged 6-12 years who attended the Pediatric Endocrinology Clinic of a tertiary care teaching hospital in Northwest India from January 2017 to March 2018 were included in the study. Obesity was defined as body mass index $(\mathrm{BMI}) \geq 2$ Z-score above the World Health Organization (WHO) 2007 Growth Reference mean. Children with stigmata of an endocrinopathy, a syndromic or a known genetic form of obesity were not considered eligible for inclusion. Thyroid dysfunction was defined as elevation of serum TSH (4-10 mIU/l) with or without alterations in total thyroxine (T4) (normal 4.5-11.5 ug/dl) or total triiodothyronine (T3) concentrations (normal 0.8-2.0 ng/ml). IsHT was defined as serum TSH level between 4 and $10 \mathrm{mIU} / \mathrm{l}$ and both T3 and T4 within the normal ranges. Children with positive thyroid autoantibodies (anti-thyroid peroxidase or antithyroglobulin) and those taking any medication that may interfere with thyroid function e.g. antiepileptics, iodinated drugs, and steroids were excluded. An informed consent from parents/ primary caregivers and assent from the child was taken before inclusion. The procedures followed during the study were in accordance with the Helsinki Declaration of 1975, as revised in 2008. The study was approved by the Institute's Ethics Committee (INT/IEC/2017/668).

\section{Anthropometric assessment}

Physical assessment included measurement of weight on electronic weighing machine (Avery, India) to the nearest 50 grams with children barefoot and wearing light clothing, and height with a stadiometer to the nearest $1 \mathrm{~mm}$. Anthropometric calculations such as weight, height and BMI Z-scores were done with WHO Anthroplus software (version 1.0.4 WHO, Geneva, Switzerland).

\section{Laboratory assessment}

Laboratory investigations included measurement of serum concentrations of total T3, total T4, TSH, total cholesterol (TC), high-density lipoprotein-cholesterol (HDL-C), and triglycerides (TG) on venous blood samples drawn from antecubital vein in the morning after overnight fasting. Thyroid hormone estimations were done by chemiluminescence assay on Advia Centaur (Siemens Healthcare Diagnostics, USA) while parameters of lipid profile were measured by using dedicated kits on Advia 1800 clinical chemistry analyzer (Siemens Healthcare Diagnostics, USA).

\section{Intervention and follow up visits}

Throughout the duration of the study, all children participated in the routine weight management intervention that consisted of behavioral modification and a diet and physical activity plan. They were assigned to one of the 2 intervention groups by a computer generated random number table. Group 1 did not receive levothyroxine while group 2 received levothyroxine in a fixed dose of $25 \mu \mathrm{g} /$ day (approximately $0.5 \mu \mathrm{g} / \mathrm{kg} /$ day). All children were followed up at 3-month intervals for 6 months. 
A detailed anthropometric assessment was done at each follow up visits in addition to the measurements of thyroid and lipid parameters. The details were recorded into a predesigned proforma.

\section{Statistical analysis}

All the baseline and follow up data was coded and entered into the excel sheet for analysis. Results were expressed as mean $\pm S D$. Baseline characteristics of the groups were compared by the Mann-Whitney $U$ test. Within group comparisons of BMI Z-score, lipid and thyroid parameters from baseline to the endpoint were made by the Wilcoxan signed rank test. The values of these parameters were compared between the groups by two sample $t$-tests. Correlations between baseline and endpoint variables were done by Spearman's correlation coefficient. All analyses were performed on statistical package for social sciences (SPSS) for Windows (Version 22.0. Armonk, NY: IBM Corp.). A $p$-value $<0.05$ was considered statistically significant.

\section{Results}

The study comprised of 51 children (33, 65\% boys) with SO. The mean age was similar in the 2 groups $(8.9 \pm 1.3$ year vs. 8.8 \pm 1.3 year). None of the children showed symptoms suggestive of hypothyroid state or had a palpable goitre. All patients had IsHT. The various anthropometric and laboratory parameters were similar in the 2 groups at baseline and at the 3-month and 6-month study time points (Table I). Within the groups, all children showed a significant decrease in weight and BMI
Z-scores, and TSH levels from baseline to the study endpoint (Table I). However, the mean decrease in these parameters was similar between the 2 groups (Table I). In addition, the mean changes in height Z-scores, total T3, total T4, TC, TG and HDL-C were also similar between the 2 groups (Table I). Normalization of $\mathrm{TSH}(<4 \mathrm{mIU} / \mathrm{l})$ occurred in 12 (48\%) of children in group 1 as compared to $15(57.6 \%)$ in group 2.

There was no significant correlation between change in BMI Z-scores to change in TSH levels in both the groups. A significant negative correlation between HDL and BMI Z-score was noted in both the groups at baseline $(r=-0.42, \mathrm{p}=0.03)$. There was a negative correlation between weight Z-score and HDL $(r=-0.47, p=0.02)$, and a positive correlation between BMI Z-score and TG $(r=0.54, p=0.007)$ at follow up visits in both the groups.

\section{Discussion}

In our study, the addition of levothyroxine to the weight reduction program did not show beneficial effects on body weight, BMI and thyroid profiles in obese children with IsHT. These results are consistent with several previous studies which demonstrated that the elevations in serum TSH in these patients respond well to the weight reduction measures and the addition of thyroxine is not needed [18-22]. In addition, levothyroxine supplementation did not show beneficial effects on the parameters of lipid profiles of these patients. Similar findings were observed by Eliakim et al. [15]. Interestingly, alterations of lipid parameters are amongst the first tissue effects of

Table I. Comparison of anthropometric and laboratory parameters between and within (change from baseline to endpoint) the 2 study groups at the 3 time points

\begin{tabular}{lllll}
\hline Variable & Time points & $\begin{array}{l}\text { Group 1 } \\
\text { mean } \pm \text { SD }\end{array}$ & $\begin{array}{l}\text { Group 2 } \\
\text { mean } \pm \text { SD }\end{array}$ & P-value \\
\hline Weight Z-score & Baseline & $3.08 \pm 1.72$ & $3.88 \pm 2.03$ & 0.14 \\
\cline { 2 - 5 } & 3-month & $2.85 \pm 1.59$ & $3.83 \pm 1.92$ & 0.06 \\
\cline { 2 - 5 } & 6-month & $2.22 \pm 1.41$ & $3.02 \pm 1.60$ & 0.07 \\
\cline { 2 - 5 } & Change, $p$-value & $-0.79 \pm 0.67,0.00$ & $-0.93 \pm 0.73,0.00$ & 0.07 \\
\hline Height Z-score & Baseline & $0.47 \pm 1.33$ & $0.74 \pm 1.17$ & 0.44 \\
\cline { 2 - 5 } & 3-month & $0.64 \pm 1.29$ & $1.03 \pm 1.25$ & 0.33 \\
\cline { 2 - 5 } & 6-month & $0.40 \pm 1.28$ & $0.78 \pm 1.28$ & 0.30 \\
\cline { 2 - 4 } & Change, $p$-value & $0.05 \pm 0.26,0.31$ & $0.01 \pm 0.34,0.83$ & 0.30 \\
\hline
\end{tabular}

T3 - triiodothyronine; T4 - thyroxine; TSH - thyroid stimulating hormone; TC - total cholesterol; TG - triglycerides, HDL-C - high-density lipoprotein cholesterol 
Table I. Comparison of anthropometric and laboratory parameters between and within (change from baseline to endpoint) the 2 study groups at the 3 time points (cont.)

\begin{tabular}{|c|c|c|c|c|}
\hline Variable & Time points & $\begin{array}{l}\text { Group } 1 \\
\text { mean } \pm \mathrm{SD}\end{array}$ & $\begin{array}{l}\text { Group } 2 \\
\text { mean } \pm \mathrm{SD}\end{array}$ & $P$-value \\
\hline \multirow[t]{4}{*}{ BMI Z-score } & Baseline & $3.69 \pm 1.31$ & $4.31 \pm 2.11$ & 0.21 \\
\hline & 3-month & $3.24 \pm 1.45$ & $4.08 \pm 1.92$ & 0.10 \\
\hline & 6-month & $2.84 \pm 1.46$ & $3.50 \pm 1.93$ & 0.19 \\
\hline & Change, $p$-value & $-0.80 \pm 0.77,0.03$ & $-0.97 \pm 0.69,0.02$ & 0.19 \\
\hline \multirow[t]{4}{*}{ Total T3 (ng/ml) } & Baseline & $2.17 \pm 0.66$ & $1.90 \pm 0.32$ & 0.41 \\
\hline & 3-month & $2.02 \pm 0.84$ & $1.93 \pm 0.39$ & 0.64 \\
\hline & 6-month & $2.25 \pm 1.04$ & $2.00 \pm 0.61$ & 0.31 \\
\hline & Change, $p$-value & $0.01 \pm 1.31,0.96$ & $0.07 \pm 0.68,0.60$ & 0.31 \\
\hline \multirow[t]{4}{*}{ Total T4 ( $\mu \mathrm{g} / \mathrm{dl})$} & Baseline & $8.92 \pm 2.4$ & $8.98 \pm 1.90$ & 0.91 \\
\hline & 3-month & $8.58 \pm 2.06$ & $9.41 \pm 1.99$ & 0.16 \\
\hline & 6-month & $8.53 \pm 1.95$ & $8.96 \pm 1.56$ & 0.40 \\
\hline & Change, $p$-value & $-0.61 \pm 1.82,0.12$ & $-0.02 \pm 1.59,0.93$ & 0.40 \\
\hline \multirow[t]{4}{*}{ TSH (mIU/l) } & Baseline & $5.51 \pm 1.00$ & $5.89 \pm 1.10$ & 0.20 \\
\hline & 3-month & $4.14 \pm 1.09$ & $3.92 \pm 1.73$ & 0.62 \\
\hline & 6-month & $4.04 \pm 1.12$ & $3.89 \pm 1.48$ & 0.69 \\
\hline & Change, $p$-value & $-1.44 \pm 1.20,0.00$ & $-1.93 \pm 1.15,0.00$ & 0.69 \\
\hline \multirow[t]{4}{*}{$\mathrm{TC}(\mathrm{mg} / \mathrm{dl})$} & Baseline & $153.0 \pm 27.2$ & $154.4 \pm 26.3$ & 0.99 \\
\hline & 3-month & $147.2 \pm 24.2$ & $144.9 \pm 22.6$ & 0.65 \\
\hline & 6-month & $142.7 \pm 22.1$ & $146.1 \pm 24.8$ & 0.95 \\
\hline & Change, $p$-value & $-10.30 \pm 6.92,0.14$ & $-8.22 \pm 7.15,0.25$ & 0.95 \\
\hline \multirow[t]{4}{*}{$\mathrm{TG}(\mathrm{mg} / \mathrm{dl})$} & Baseline & $127.6 \pm 44.2$ & $122.5 \pm 49.8$ & 0.80 \\
\hline & 3-month & $124.0 \pm 54.6$ & $115.2 \pm 43.4$ & 0.65 \\
\hline & 6-month & $126.2 \pm 35.5$ & $112.1 \pm 36.6$ & 0.15 \\
\hline & Change, $p$-value & $-2.34 \pm 34.57,0.74$ & $-15.72 \pm 40.69,0.07$ & 0.15 \\
\hline \multirow[t]{4}{*}{ HDL-C (mg/dl) } & Baseline & $39.6 \pm 6.2$ & $42.3 \pm 9.7$ & 0.18 \\
\hline & 3-month & $38.8 \pm 6.3$ & $40.0 \pm 7.5$ & 0.53 \\
\hline & 6-month & $40.8 \pm 6.6$ & $41.8 \pm 7.8$ & 0.67 \\
\hline & Change, $p$-value & $0.85 \pm 6.17,0.51$ & $-0.71 \pm 10.03,0.73$ & 0.67 \\
\hline
\end{tabular}

T3 - triiodothyronine; T4 - thyroxine; TSH - thyroid stimulating hormone; TC - total cholesterol; TG - triglycerides, HDL-C - high-density lipoprotein cholesterol 
a hypothyroid state and several studies in adults have shown that treatment with levothyroxine has clear benefits on TC and LDL-C even in patients with mild SCH [23]. This beneficial effect of levothyroxine on lipid abnormalities is one of the main arguments that favours treatment of patients with $\mathrm{SCH}$ [24]. Similar to adult patients, mild $\mathrm{SCH}$ in children and adolescents has also been found to be associated with increases in TC and nonHDL cholesterol $[25,26]$. But it is not known if specific treatment of mild SCH in children leads to improvement in dyslipidemia [26, 27]. Large randomized placebo controlled studies are therefore needed to determine if treatment of mild SCH in obese children leads to improvement in lipid parameters [26].

The data on the effects of specific therapy of ISHT with levothyroxine in children with $\mathrm{SO}$ is scarce [4]. A majority of the studies of levothyroxine supplementation conducted on non-obese children with IsHT, were not focused on changes in body weight or BMI [28]. One longitudinal study on comparative evaluation of 2-year therapy with levothyroxine versus no treatment in non-obese children with mild $\mathrm{SCH}$, did not find any significant difference between the two groups in terms of BMI Z-scores [29]. Forty two of the 69 children who normalized their TSH after withdrawal of levothyroxine did not show any changes in BMl during the 2-year period indicating that the effects of addition of levothyroxine on anthropometric parameters were negligible [29].

In children with $\mathrm{SO}$, we could find only 2 previous studies on levothyroxine treatment of their IsHT $[15,16]$. Eliakim et al. from Israel studied the effect of levothyroxine supplementation on body weight, linear growth and lipid profiles in 196 obese children and adolescents [15]. Fifteen of the obese children with IsHT also received levothyroxine substitution for 6 months and were compared to 26 non-treated subjects [15]. Treatment with levothyroxine showed no significant effect on body weight, linear growth and lipid profile except for a greater decrease in serum TG levels [15]. Serum TSH concentrations returned to normal ranges in the majority of children with IsHT who participated in the combined intervention irrespective of levothyroxine treatment [15]. In a more recent retrospective study, Matusik et al. from Poland analyzed the changes in anthropometrical (delta BMI Z-score) and hormonal (delta TSH) status from baseline to the first follow-up visit in 51 obese children with IsHT who were treated according to the same weight reduction program [16]. The proportion of children who showed normalization of TSH was similar in the group (25 children) that received additional levothyroxine as compared to the group (26 children) that did not receive levothyroxine (90.5\% vs. $80.9 \%$ respectively) [16]. The difference in the delta BMI Z-scores between the 2 groups was also insignificant. The study demonstrated that dietary- behavioral management intervention contributed to reduction of BMI irrespective of levothyroxine use in obese children with IsHT [16]. It concluded, quite similar to our study that elevated levels of TSH are a consequence rather than cause of overweight and pharmacological treatment should be avoided [16].

The usual dose of levothyroxine in children with $\mathrm{SCH}$ is $2 \mu \mathrm{g} / \mathrm{kg} /$ day $[28,29]$. We chose a low dose of levothyroxine in view of our general observation of a mild IsHT in children with SO and observations by others that even a small dose in these patients might occasionally cause mild hyperthyroidism $[16,28]$. None of our patients showed clinical or biochemical evidence of hyperthyroidism.

Although our study is the first prospective evaluation of specific treatment of ISHT in children with SO, we realize the limitations of a small sample size, use of total and not the free hormone concentrations, lack of blinding and an observation period not long enough to draw definite conclusions. It nevertheless provides a basis for further prospective, larger, wellcontrolled clinical trials to elucidate the role of levothyroxine in an ever-increasing population of children with SO. In addition, there are several areas in obesity associated thyroid dysfunction which require further research [4]. For example, it is not fully known if addition of levothyroxine to the weight loss interventions has additional benefits on body composition, energy expenditure, insulin sensitivity, lipid and glucose metabolism, NAFLD as well as other cardiometabolic factors such as blood pressure and cardiac status [4, 30]. It is thought that the elevation of TSH in obese children and adolescents might have a negative influence on the cardiac status due to its association with a higher TC and blood pressure [31]. Only longitudinal studies can confirm this hypothesis and establish whether a substitutive treatment with levothyroxine is really needed in these patients [31]. A recent systematic review suggests that levothyroxine replacement therapy can reduce blood pressure in adults with $\mathrm{SCH}$ but the results need to be validated in more clinical trials with larger samples [32]. Similar trials are also needed in obese children with IsHT. Recent data also indicates that thyroid hormones induce browning of the white adipose tissue and thus may help in nullifying the effects of extra energy intake [33]. This may have clinical implications for children and adults with SO not only by enabling them to stay slim but also counteracting the comorbidities such as type 2 diabetes [34].

In conclusion, the addition of levothyroxine to weight management interventions has no beneficial effect on reduction in $\mathrm{BMI}$ and parameters of lipid and thyroid profiles in children with obesity. Larger, longitudinal trials of levothyroxine supplementation are needed to ascertain the cardiometabolic benefits in children with thyroid dysfunction due to simple obesity. 


\section{References}

1. Greydanus DE, Agana M, Kamboj MK, et al. Pediatric obesity: Current concepts. Dis Mon 2018; 64: 98-156. doi: 10.1016/j.disamonth.2017.12.001

2. Dayal D, Jain H, Attri SV, et al. Relationship of high sensitivity C-reactive protein levels to anthropometric and other metabolic parameters in Indian children with simple overweight and obesity. J Clin Diagn Res 2014; 8: PC05-8. doi: 10.7860/JCDR/2014/8191.4685

3. Fontenelle LC, Feitosa MM, Severo JS, et al. Thyroid function in human obesity: Underlying mechanisms. Horm Metab Res 2016; 48: 787-794. doi: 10.1055/s-0042-121421

4. Yadav J, Jain N, Dayal D. Alterations of thyroid function in overweight and obese children: An update. Indian J Child Health 2018; 5: 145-150. doi: 10.5603/EP.2017.0007

5. Dayal D, Kumar TS. High prevalence of thyroid dysfunction in North Indian children with simple obesity. Indian Pediatr 2019; 56: 331.

6. Wasniewska M, Aversa T, Salerno M, et al. Five-year prospective evaluation of thyroid function in girls with subclinical mild hypothyroidism of different etiology. Eur J Endocrinol 2015; 173: 801-808. doi: 10.1530/EJE-15-0484

7. Aversa T, Valenzise M, Corrias A, et al. Underlying Hashimoto's thyroiditis negatively affects the evolution of subclinical hypothyroidism in children irrespective of other concomitant risk factors. Thyroid 2015; 25: 183-187. doi: 10.1089/thy.2014.0235

8. De Luca F, Wasniewska M, Zirilli G, et al. At the end of a two-year follow-up elevated TSH levels normalize or remain unchanged in most the children with subclinical hypothyroidism. Ital J Pediatr 2010; 36: 11. doi: 10.1186/1824-7288-36-11

9. Arrigo T, Wasniewska M, Crisafulli G, et al. Subclinical hypothyroidism: the state of the art. J Endocrinol Invest 2008; 31: 79-84. doi: 10.1007/BF03345571

10. Crisafulli G, Aversa T, Zirilli G, et al. Subclinical Hypothyroidism in Children: When a Replacement Hormonal Treatment Might Be Advisable. Front Endocrinol (Lausanne) 2019; 10: 109. doi: 10.3389/ fendo.2019.00109

11. Sert A, Pirgon O, Aypar E, et al. Subclinical hypothyroidism as a risk factor for the development of cardiovascular disease in obese adolescents with nonalcoholic fatty liver disease. Pediatr Cardiol 2013; 34: 1166-1174. doi: 10.1007/s00246-013-0638-z

12. Cerbone M, Capalbo D, Wasniewska M, et al. Cardiovascular risk factors in children with long-standing untreated idiopathic subclinical hypothyroidism. J Clin Endocrinol Metab 2014; 99: 2697-2703. doi: 10.1210/jc.2014-1761

13. Kaltenbach TE, Graeter T, Oeztuerk S, et al. Thyroid dysfunction and hepatic steatosis in overweight children and adolescents. Pediatr Obes 2017; 12: 67-74. doi: 10.1111/ijpo.12110

14. Witkowska-Sędek E, Kucharska A, Rumińska M, et al. Thyroid dysfunction in obese and overweight children. Endokrynol Pol 2017; 68: 54-60. doi: 10.5603/EP.2017.0007

15. Eliakim A, Barzilai M, Wolach B, et al. Should we treat elevated thyroid stimulating hormone levels in obese children and adolescents? Int J Pediatr Obes 2006; 1: 217-221.

16. Matusik P, Gawlik A, Januszek-Trzciakowska A, et al. Isolated subclinical hyperthyrotropinemia in obese children: Does levothyrox- ine (LT4) improve weight reduction during combined behavioral therapy? Int J Endocrinol 2015; 2015: 792509. doi: 10.1155/2015/ 792509

17. Kaptein EM, Beale E, Chan LS. Thyroid hormone therapy for obesity and nonthyroidal illnesses: A systematic review. J Clin Endocrinol Metab 2009; 94: 3663-3675. doi: 10.1210/jc.2009-0899

18. Reinehr T, de Sousa G, Andler W. Hyperthyrotropinemia in obese children is reversible after weight loss and is not related to lipids. J Clin Endocrinol Metab 2006; 91: 3088-3091. doi: 10.1210/jc. 2006-0095

19. Reinehr T, Andler W. Thyroid hormones before and after weight loss in obesity. Arch Dis Child 2002; 87: 320-323. doi: 10.1136/ adc.87.4.320

20. Marras V, Casini MR, Pilia S, et al. Thyroid function in obese children and adolescents. Horm Res Paediatr 2010; 73: 193-197. doi: 10.1159/000284361

21. Grandone A, Santoro N, Coppola F, et al. Thyroid function derangement and childhood obesity: An Italian experience. BMC Endocr Disord 2010; 10: 8. doi: 10.1186/1472-6823-10-8

22. Aeberli I, Jung A, Murer SB, et al. During rapid weight loss in obese children, reductions in TSH predict improvements in insulin sensitivity independent of changes in body weight or fat. J Clin Endocrinol Metab 2010; 95: 5412-5418. doi: 10.1210/jc.2010-1169

23. Li X, Wang Y, Guan Q, et al. The lipid-lowering effect of levothyroxine in patients with subclinical hypothyroidism: A systematic review and meta-analysis of randomized controlled trials. Clin Endocrinol (Oxf) 2017; 87: 1-9. doi: 10.1111/cen.13338

24. Duntas LH, Brenta G. A Renewed Focus on the Association Between Thyroid Hormones and Lipid Metabolism. Front Endocrinol (Lausanne) 2018; 9: 511. doi: 10.3389/fendo.2018.00511

25. Dayal D, Saini L, Attri SV, et al. Daily versus alternate day thyroxine therapy to maintain euthyroidism in children with congenital hypothyroidism. Int J Endocrinol Metab 2013; 11: e9499. doi: 10.5812/ ijem.9499

26. Dahl AR, Iqbal AM, Lteif AN, et al. Mild subclinical hypothyroidism is associated with paediatric dyslipidaemia. Clin Endocrinol (Oxf) 2018; 89: 330-335. doi: 10.1111/cen.13752

27. Cerbone M, Capalbo D, Wasniewska M, et al. Effects of L-thyroxine treatment on early markers of atherosclerotic disease in children with subclinical hypothyroidism. Eur J Endocrinol 2016; 175: 1119. doi: 10.1530/EJE-15-0833

28. Vigone MC, Capalbo D, Weber G, et al. Mild Hypothyroidism in Childhood: Who, When, and How Should Be Treated? J Endocr Soc 2018; 2: 1024-1039. doi: 10.1210/js.2017-00471

29. Wasniewska M, Corrias A, Aversa T, et al. Comparative evaluation of therapy with L-thyroxine versus no treatment in children with idiopathic and mild subclinical hypothyroidism. Horm Res Paediatr 2012; 77: 376-381. doi: 10.1159/000339156

30. Zhao T, Chen B, Zhou Y, et al. Effect of levothyroxine on the progression of carotid intima-media thickness in subclinical hypothyroidism patients: a meta-analysis. BMJ Open 2017; 7: e016053. doi: 10.1136/bmjopen-2017-016053

31. Radetti G, Grugni G, Lupi F, et al. The relationship between hyperthyrotropinemia and metabolic and cardiovascular risk factors in a large group of overweight and obese children and adolescents. 
J Endocrinol Invest 2017; 40: 1311-1319. doi: 10.1007/s40618017-0705-z

32. He W, Li S, Zhang JA, et al. Effect of levothyroxine on blood pressure in patients with subclinical hypothyroidism: a systematic review and meta-analysis. Front Endocrinol (Lausanne) 2018; 9: 454. doi: 10.3389/fendo.2018.00454
33. Martínez-Sánchez N, Moreno-Navarrete JM, Contreras C, et al. Thyroid hormones induce browning of white fat. J Endocrinol 2017; 232: 351-362. doi: 10.1530/JOE-16-0425

34. Weiner J, Hankir M, Heiker JT, et al. Thyroid hormones and browning of adipose tissue. Mol Cell Endocrinol 2017; 458: 156-159. doi: 10.1016/j.mce.2017.01.011 